\title{
La Modelización peatonal como herramienta para la mejora del espacio urbano
}

\author{
José Luis López Oliete ${ }^{1}$ \\ Ing. Caminos, Canales y Puertos, Consultor de Transportes en ARUP, España
}

Raquel Goyanes López

Licenciada en Geografía, Consultora Senior de Transportes en ARUP, España

Rafael Ruiz López

Ing. Caminos, Canales y Puertos, Responsable del Área de Tráfico y Movilidad en ARUP, España

\section{RESUMEN}

El valor y la conveniencia de un espacio urbano está determinado en gran medida por su carácter, y las calles juegan un papel clave en la determinación de cómo las personas experimentan e interactúan con su entorno. La vida urbana tiene que ver con las personas. Su presencia a lo largo de las calles y otros espacios públicos es el principal indicador de un entorno urbano vibrante. La forma en la que está dispuesto el espacio y cómo se relaciona con los usos del suelo establecidos en él, tiene una incidencia significativa en el comportamiento peatonal.

La planificación del movimiento de las personas es un factor clave en el éxito del diseño de un espacio urbano, ya sea al aire libre o de un edificio. Es esencial comprender la dinámica de flujos, analizar los requisitos de capacidad para la circulación y acumulación de espacios y encontrar una lógica del comportamiento peatonal ante las alteraciones de su entorno espacial, ya sean grandes o pequeñas.

La simulación peatonal, a través de programas especializados, demuestra ser una herramienta práctica que aporta valiosa información para la planificación y proceso de diseño de nuevos espacios peatonales urbanos o la mejora de los existentes. La capacidad de simulación creciente reduce el tiempo de análisis, y la creciente simplicidad de uso amplía el alcance de proyectos más allá de costosos análisis de evacuaciones de edificios altos, estaciones, estadios, etc.

\section{INTRODUCCIÓN}

Desde hace miles de años el ser humano diseña y construye ciudades, que albergan espacios públicos que sirven para lugar de reunión, celebración y estancia de la comunidad. Los griegos tenían el ágora y los teatros, mientras que los romanos el foro, los circos y otras edificaciones públicas como las basílicas, en los cuales el acceso y dispersión de los espectadores y visitantes se lograban en tiempos razonablemente cortos. Con el acervo constructivo desarrollado por estos pueblos, la civilización occidental heredó de estos el arte

\footnotetext{
${ }^{1}$ Autores: jose.lopez@arup.com; raquel.goyanes@arup.com; rafael.ruiz@arup.com Tel.: +34 915239276 c/ Alcalá, 54. Madrid (28014), España.
} 
de hacer ciudades, diseñando de forma aparentemente razonable los elementos que dotan de accesos a los distintos espacios integrados en la trama urbana. John Fruin, que con su estudio, recogido en el libro Pedestrian Planning and Design (1971), puso las bases del análisis y modelización peatonal, menciona que, antes de la civilización grecolatina, una de las primeras referencias escritas sobre el dimensionamiento de los accesos a edificios públicos se puede encontrar en el Talmud, el libro de las leyes del judaísmo, y que en las construcciones egipcias se observaba la repetición y desarrollo de diversos elementos arquitectónicos que ponen de relieve su conocimiento y estudio del problema.

Sin embargo, la tendencia cada vez más creciente de concentración de población en las ciudades y el aumento de la población mundial hacen que hoy en día se busquen nuevas respuestas al desafío que supone densidades urbanas cada vez mayores, alejadas de los estándares tradicionales de concepción del espacio público, basado en proporciones y usos heredados de épocas anteriores.

En esta ponencia se analizan en un primer lugar los desafíos a los que se tiene que hacer frente durante el proceso de diseño de espacios urbanos concebidos para acoger grupos de personas, desde varias a cientos de miles. Se mencionan las diferencias entre un enfoque estático del problema y una modelización dinámica, incluyendo una breve aproximación teórica a los modelos de simulación peatonal, y la presentación de varios proyectos de espacios urbanos efectuados por Arup en los que la simulación peatonal fue un elemento clave para la validación del diseño. Para ello, Arup utilizó su propio software de simulación peatonal, MassMotion, que es una de las herramientas más avanzadas del mundo en la predicción de los flujos peatonales en entornos complejos tridimensionales, y que permite analizar los resultados de la modelización mediante la visualización de animaciones en tiempo real y el tratamiento numérico de los datos de salida procedentes del modelo.

\section{DISEÑO DEL ESPACIO URBANO DESDE UN ENFOQUE ESTÁTICO}

El espacio urbano se diseña con el fin último de servir a las personas como lugar donde poder interactuar, reunirse o moverse. Sin embargo, muchas veces después de que dichos espacios hayan sido construidos y abiertos al público se descubre que, aun cumpliendo el marco normativo, no se alcanzan los objetivos para los que estos espacios fueron concebidos. Posibles actuaciones correctoras o mitigadoras sobre esa realidad ya construida suponen un incremento adicional de los costes del proyecto, que muchas veces no pueden acometerse bien por motivos técnicos o económicos. A este respecto, una gran parte de los profesionales que trabajan en el diseño de espacios urbanos y edificios de uso público muestran, por lo general, una significativa dependencia normativa durante el proceso de planificación y estudio de los flujos peatonales en dichos espacios, abordando el problema generalmente desde una óptica determinista. Esta forma de entender el problema da lugar a un dimensionamiento y configuración del espacio urbano basado fundamentalmente en la aplicación de estándares internacionales y ratios cuyo origen es empírico, pero que han sido obtenidos en unas condiciones muy específicas que no varían en el tiempo, lo que se entiende como análisis estático. 
La siguiente tabla muestra algunos de los ratios de capacidad más utilizados en el análisis estático o determinista para el dimensionamiento de algunos elementos de espacios públicos con una importante afluencia de personas (estaciones de tren, estadios).

\begin{tabular}{|c|c|c|c|}
\hline Elemento & Capacidad & Unidades & Estándar internacional \\
\hline Tornos de acceso & 660 & personas/torno y hora & \multirow{3}{*}{$\begin{array}{l}\text { Guide to Safety at Sport Grounds } \\
\text { (Reino Unido) }\end{array}$} \\
\hline Superficie plana & 82 & personas/min y metro & \\
\hline Escaleras & 66 & personas/min y metro & \\
\hline Escaleras mecánicas & 120 & personas/min & $\begin{array}{l}\text { London Underground Station Standards } \\
\text { (Reino Unido) }\end{array}$ \\
\hline
\end{tabular}

Tabla 1 - Algunos estándares internacionales habituales en el enfoque estático.

Sin embargo, dada la aleatoriedad propia del comportamiento humano y de la imposibilidad de determinar con exactitud cómo, cuándo y hacia dónde se producen los flujos peatonales, la resolución del problema por medio de este enfoque requiere generalmente de la inclusión de un sinfín de hipótesis adicionales que acoten los flujos esperados por elemento en función de las distintas alternativas de acceso y dispersión peatonal existentes en el entorno construido. Sin embargo, estas alternativas suelen ser muy numerosas, cambiantes con el tiempo y el nivel de demanda, difíciles de precisar en la mayoría de casos, por lo que es necesario recurrir a la simplificación del problema mediante la inclusión de hipótesis, lo que induce arbitrariedad y cierto sesgo en función de quién las efectúe, conduciendo finalmente a la elección de soluciones constructivas que en muchos casos distan de ser las adecuadas.

Los estándares generalmente utilizados para el dimensionamiento de ciertos elementos urbanos no permiten cuantificar el impacto sobre el comportamiento peatonal que tendría una posible alteración de estos o de otros componentes del entorno espacial, ni tampoco recoge otros fenómenos aleatorios que a menudo se producen diariamente en el ámbito urbano. En este sentido, y como ejemplo de lo anterior, el impacto producido por la avería de un tramo de escaleras mecánicas en los accesos de una estación de tren durante la hora punta podría dar lugar a comportamientos inesperados del flujo peatonal, con una redistribución y concentración de dichos flujos en otras zonas en las que se podrían alcanzar densidades elevadas que empeorarían significativamente los niveles de servicio. Incluso pondría en situación de peligro a los usuarios de la estación, si dicha situación no se prevé y no se estudia convenientemente.

Además, el análisis estático o determinista no permite representar adecuadamente el comportamiento de un grupo formado por varias personas que se mueven al mismo tiempo en un espacio compartido, en los que existe además una componente adicional de respuesta colectiva concomitante a la decisión racional de cada individuo. Estas limitaciones metodológicas requieren abordar el problema de otro modo, desde una óptica estocástica o dinámica, que reproduzca el libre albedrío sobre el que se basa la lógica del comportamiento humano e incluya la componente grupal.

En este sentido, el avance de la técnica en las últimas décadas permite disponer actualmente de ordenadores potentes que realizan en muy poco tiempo complejos análisis dinámicos, 
mediante el uso de programas informáticos que incluyen en sus algoritmos componentes aleatorias que simulan con gran realismo el movimiento de flujos peatonales, grandes o pequeños, en espacios tridimensionales. Las geometrías de dichos espacios se pueden alterar a conveniencia durante su fase de planificación y diseño, creando diferentes escenarios y diversos tipos de usuarios, permitiendo evaluar multitud de soluciones con todo detalle antes de su construcción, bajo unos parámetros óptimos de seguridad y comodidad para las personas potencialmente usuarias de dicho espacio público.

\section{BREVE APROXIMACIÓN TEÓRICA A LA MODELIZACIÓN PEATONAL}

La mayor parte de los estudios y cálculos de modelización peatonal, tanto estática como dinámica, tienen su base en el trabajo elaborado por John Fruin y recogidos en su libro Pedestrian Planning and Design (1971). Tal y como se ha comentado con anterioridad, el notable desarrollo de la técnica informática y programas específicos avanzados permite modelizar con gran realismo el movimiento de grandes flujos peatonales en espacios tridimensionales con el objetivo de asegurar la comodidad y la seguridad de los usuarios de dichos espacios.

La modelización peatonal (crowd motion en inglés), considera a cada peatón como una partícula newtoniana que decide el camino o ruta hacia su destino de forma racional. Esta elección responde tanto a fuerzas de carácter físico como social, incluyendo modelos numéricos que tienen el objetivo de reproducir de la manera más realista posible el comportamiento colectivo de la multitud. El proceso de elección de la ruta a seguir por el usuario se puede asimilar al coste generalizado que este percibe de cada una de las rutas disponibles, intentando minimizar dicho coste. El algoritmo del coste total de la ruta seguida por un usuario (i) se podría representar con la siguiente expresión:

$$
C_{\text {ruta } i}=W_{D} \cdot\left(\frac{D}{v}\right)+W_{Q} \cdot Q+W_{E} \cdot E
$$

En la anterior expresión $D$ denota la distancia total a recorrer por un peatón desde el origen de su viaje hasta su destino, $v$ es la velocidad deseada de desplazamiento del peatón, $Q$ es el tiempo de espera estimado antes de acceder al elemento de conexión entre dos áreas (entrada, rampa, escalera, torno de acceso, etc.), y $E$ es el coste asociado a cada tipo de elemento de conexión anteriormente mencionado. Es importante destacar que cada término del algoritmo del coste de la ruta está multiplicado por un peso aleatorio $\left(W_{D}, W_{Q}, W_{E}\right)$, con el objetivo de representar en el coste generalizado percibido en función del tipo de usuario y sus patrones de comportamiento (tiempos de espera admisibles, velocidad deseada, distancias a recorrer, espacio necesario para caminar, etc.).

De cara a evaluar los potenciales problemas de congestión, se estima como densidad máxima admisible un ratio de cuatro personas por metro cuadrado, en aquellos espacios urbanos que presenten alta afluencia y densidad de personas, como recintos deportivos o infraestructuras 
de transporte. Una vez que se calculan las densidades para cada área y se representan en mapas y gráficos bidimensionales, se calculan los niveles de servicio de Fruin, que oscilan entre el A (óptimo) y el F (pésimo, colapso de la red), de acuerdo con las condiciones de confort percibidas por los usuarios a lo largo de sus desplazamientos.
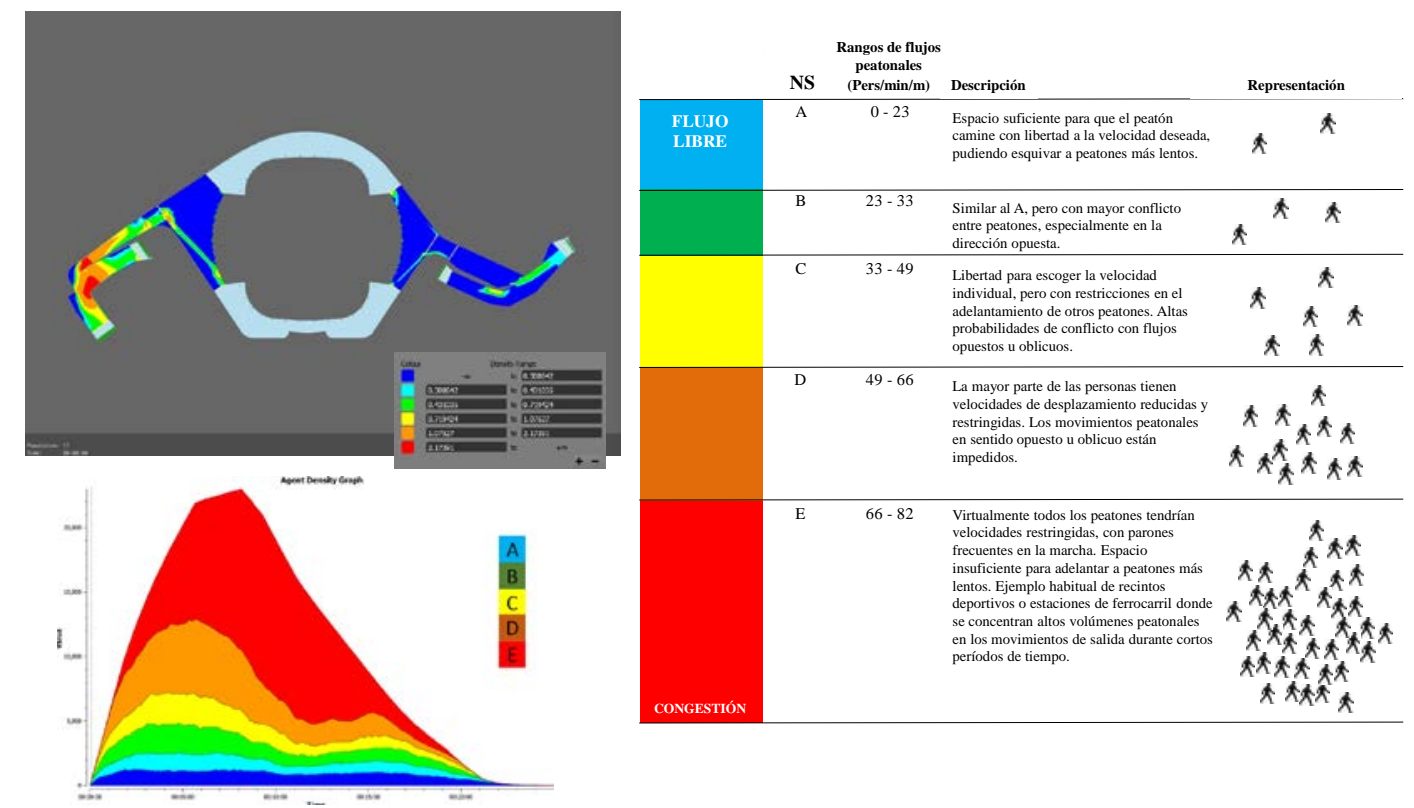

Fig. 1 - Densidades y NS con MassMotion en un estadio de fútbol (Arup).

La calibración y validación del modelo se basa en información variada que abarca desde la definición de distribuciones estadísticas de la velocidad de cada tipo de usuario en los diferentes elementos del espacio urbano (escaleras, rampas, etc.), hasta los niveles de flujo peatonal en función de las densidades alcanzadas, de acuerdo con los Niveles de Servicio definidos por Fruin para cada tipo de elemento (superficies planas, escaleras o colas).

\section{DISEÑO DEL ESPACIO URBANO BAJO UN ENFOQUE DINÁMICO}

Tal y como se comentaba en apartados precedentes, el análisis dinámico de varias soluciones geométricas posibles para un espacio público permite identificar la configuración y localización óptima de sus elementos más significativos, como escaleras mecánicas, puertas, ascensores, o tornos de acceso en estaciones de metro, estadios o edificios en altura. Estos elementos, que son lugares de conexión de un espacio público con otro contiguo, actúan como impedancias del sistema que ralentizan, y en algunos casos interrumpen, el flujo peatonal, generando incomodidad en los usuarios cuando se alcanzan determinados niveles de servicio. Es por ello por lo que su estudio y análisis detallado resulta fundamental para que el lugar público funcione correctamente, concebido y entendido como suma de múltiples espacios adyacentes.

A este respecto, se ha comprobado que con ligeras modificaciones geométricas de estos elementos o con su posible reubicación, se consigue aumentar notablemente la capacidad de estos y por ende, la comodidad de los usuarios del espacio público correspondiente. Por 
ejemplo, la disposición espacial de una hilera de tornos de acceso en un estadio y su ángulo relativo respecto al flujo peatonal entrante puede hacer variar notablemente su capacidad, y por consiguiente, reducir el tiempo de espera por usuario para entrar en el recinto. El cambio de ubicación de las escaleras mecánicas en un centro comercial o en un intercambiador de transportes también puede dar lugar a mejoras significativas en el comportamiento global de los flujos peatonales existentes en dicho espacio, aumentando la comodidad y mejorando la percepción del usuario en la medida en que este no encuentra obstáculos en su camino y puede caminar a la velocidad que estime oportuna.

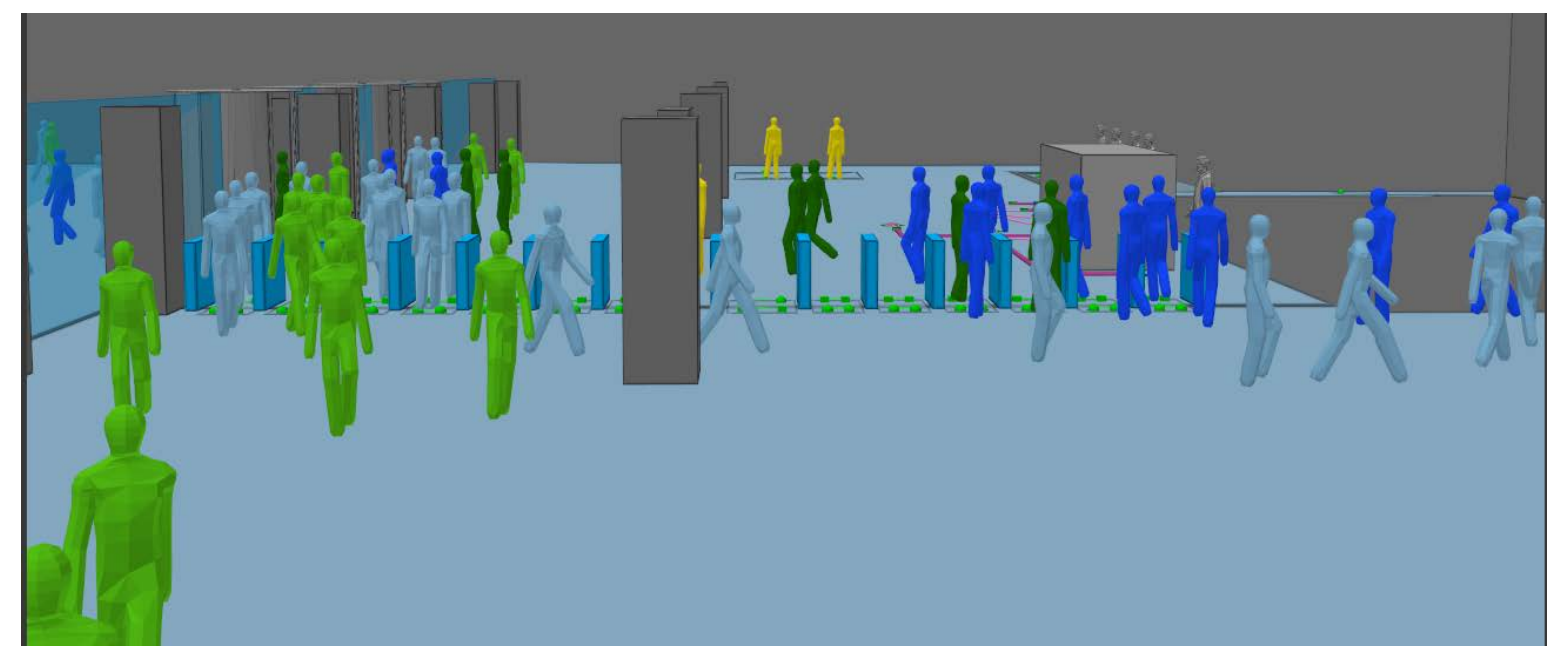

Fig. 2 - Modelización con MassMotion del acceso a un edificio de oficinas (Arup).

La imagen de la figura anterior muestra la modelización peatonal del acceso a un edificio de oficinas en Nueva York desarrollado por Arup en 2015 haciendo uso de MassMotion. El trabajo tenía el objetivo de analizar el cambio de sede de una empresa local a un rascacielos de oficinas, construyendo para ello un modelo peatonal con el nuevo diseño propuesto para el lobby del edificio, y evaluando dicho espacio desde el punto de vista de la experiencia del usuario. El modelo incluía las áreas de circulación general del vestíbulo principal, los tornos de acceso al vestíbulo, escaleras, ascensores y zona de recepción. El modelo de MassMotion permitió identificar el número de tornos de acceso que eran necesarios para garantizar una serie de tiempos máximos de espera, y las colas que se producían en los ascensores, entre otros.

Por otro lado, la diferenciación entre espectadores habituales de un evento deportivo, que conocen perfectamente el recorrido hacia el estadio así como los modos de acceso, y los nuevos espectadores que necesitan más tiempo para completar el trayecto hasta sus asientos, permitiría definir distintas distribuciones de acceso y no sobreestimar las capacidades en algunas secciones en las que podrían aparecer problemas de capacidad derivados de la existencia de usuarios que no conocen el camino y no toman correctamente sus decisiones. Esta diferenciación entre espectadores o usuarios también permite incluir diversos perfiles de comportamiento (por ejemplo, más o menos agresivos), el espacio que necesitan para caminar (por ejemplo, pasajeros con equipaje en un aeropuerto, que requieren mayor área 
para moverse), o la velocidad a la que se desplazan (personas mayores, PMR, etc.), y que influye en la capacidad de circulación del espacio público analizado.

El análisis dinámico también ayuda a definir diseños que minimicen los tiempos de espera en la formación de colas (y que no colapsen el resto de la instalación), como por ejemplo en el control de seguridad de los aeropuertos, en los mostradores de facturación, o en las taquillas de un museo. El funcionamiento de las colas viene determinado por el comportamiento de distintos tipos de visitantes, en los que la percepción subjetiva de cada individuo en relación a los tiempos de espera y las distancias de recorrido, puede modificar sustancialmente cómo acceden finalmente estos y su experiencia global en ese espacio urbano en concreto.

La aplicación del análisis dinámico y la correspondiente simulación peatonal no elimina la necesidad de realizar cálculos estáticos, dado que estos se utilizan como una validación preliminar de los diseños en su fase inicial, en donde se comprueba que las geometrías propuestas cumplen con la normativa aplicable y los estándares de referencia. Una vez comprobados dichos estándares, se procedería a efectuar el análisis dinámico del diseño propuesto, llegando en su caso a su validación final si se obtiene un resultado positivo en términos de comodidad y seguridad para los usuarios. En caso de existencia de anomalías o disconformidades se procedería de forma iterativa a repetir el proceso desde el inicio con los nuevos diseños producidos (análisis estático), tal y como muestra la siguiente imagen.

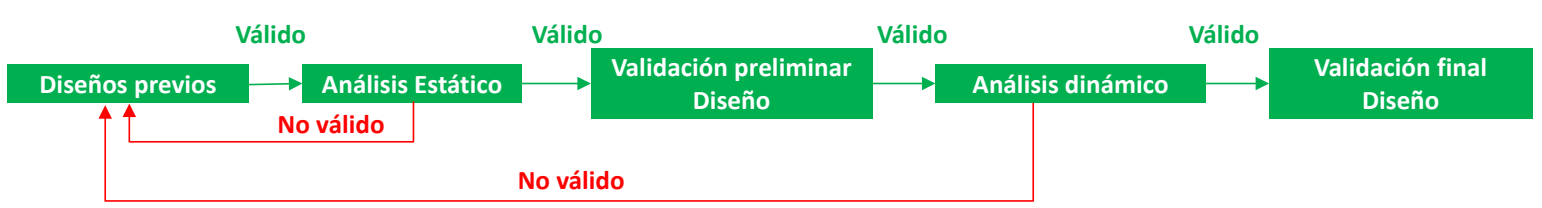

Fig. 3 - Proceso de validación preliminar y final del diseño de un espacio urbano.

Es por ello por lo que la simulación peatonal se convierte en una herramienta fundamental de validación de distintas alternativas de diseño para un mismo espacio público, pero es importante tener en cuenta que no es una herramienta de diseño en sí, ni que tampoco se elimina la necesidad de efectuar previamente algunas hipótesis y cálculos estáticos.

En el caso de los recintos deportivos, el gran flujo atraído de espectadores puede dar lugar a enormes problemas de accesibilidad y de movilidad que el entorno urbano contiguo muchas veces es incapaz de absorber. En estos casos es necesario efectuar el análisis detallado de los enormes flujos peatonales atraídos, mostrando perfectamente los puntos de mayor congestión sobre el espacio urbano diseñado, permitiendo redefinir geométricamente algunos de los espacios públicos concebidos previamente. También es necesario identificar los períodos en los que es más probable que dichos problemas de congestión tengan lugar, dado que el perfil de distribución horaria varía en función del día de la semana, y los flujos no son siempre los mismos ni las personas se comportan de igual modo. La simulación peatonal permite modelizar muchos escenarios distintos y bajo distintas hipótesis, en un intervalo de tiempo razonable y con un coste mínimo. 

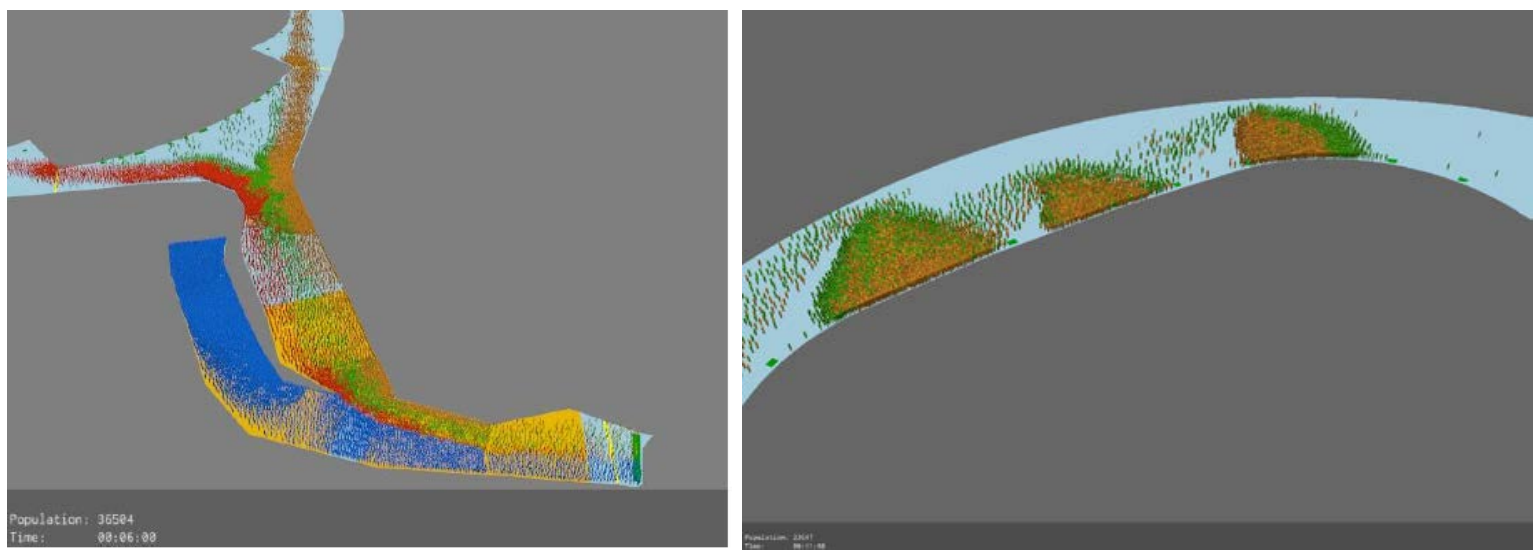

Fig. 4 - Modelización peatonal con MassMotion de un estadio de fútbol (Arup).

Por ejemplo, la normativa comúnmente aplicable en el diseño de grandes infraestructuras para acoger espectáculos deportivos, como los estadios de fútbol, requiere que el estadio sea evacuado en un corto período de tiempo, por lo que un análisis dinámico correcto puede ayudar a identificar los cambios necesarios en la geometría o el diseño para cumplir con los requisitos de organismos como FIFA.

Las imágenes de la anterior figura responden a la modelización peatonal del diseño exterior de un estadio de fútbol realizado por Arup en 2015. El trabajo consistió en el desarrollo de un modelo preliminar basado en la arquitectura de la fase de anteproyecto. El diseño preliminar del estadio incluía una gradas de accesos por lado este y oeste del estadio. En este modelo se evaluó tanto el ingreso y salida de espectadores en una situación normal de partido, como en un escenario de evacuación en el entorno del estadio, cumpliendo con los estándares que marca la FIFA y otras guías de referencia como la Green Guide. Los resultados del modelo sirvieron para orientar al equipo de diseño en el trazado y dimensionamiento de las vías de acceso exteriores del estadio. En este sentido, los resultados iniciales obtenidos a partir de un modelo estático identificaron de forma preliminar varios puntos de conflicto donde la capacidad de los accesos se sobrepasaba y daba lugar a importantes niveles de congestión y formación de colas. Los tiempos de evacuación se encontraban en el límite que garantizaba la seguridad de los espectadores. Por otro lado, la modelización dinámica peatonal con MassMotion tuvo como objeto verificar si la configuración de las rampas de acceso al estadio permitía la correcta evacuación tanto en situación normal como de emergencia. Para ello, se crearon varios modelos de demanda, con distintos perfiles de usuarios, así como curvas de llegada/salida, etc. Los resultados de los modelos identificaron que el diseño propuesto originaba puntos de aglomeración de personas y que una de las rampas de acceso no garantizaba la absorción del flujo de peatones que por ella debía circular, y que por lo tanto, no se aseguraba el cumplimiento de los parámetros de seguridad marcados por la FIFA.

Como se ha comentado con anterioridad, el análisis dinámico también se utiliza para la evaluación de infraestructuras de transporte, donde se producen grandes aglomeraciones de 
viajeros en ciertos períodos del día. La siguiente figura corresponde a la modelización peatonal de un intercambiador de transporte en Holanda, efectuado por Arup en 2016 mediante MassMotion. El modelo está basado en la arquitectura de la fase de diseño y planificación del proyecto de ampliación y rediseño de un edificio que proporciona acceso a las líneas de tren, metro, tranvía y bus. En este modelo se buscó determinar cuál era la alternativa de diseño óptima para el viajero. A este respecto, la modelización dinámica en MassMotion permitió la comprobación de distintas alternativas de localización de los tornos de validación, pudiendo definir eventos y acciones complejas que los pasajeros realizan en la realidad y que ralentizan el flujo peatonal, reproduciéndolos detalladamente en la simulación, como pudieran ser el proceso de compra de un billete o la validación del mismo antes de acceder.

A partir de mapa de densidades se localizaron puntos con elevadas densidades de personas, en andenes o escaleras mecánicas, para lo que se propusieron medidas de mitigación, que incluyeron la ampliación de andenes o el cambio en los horarios.

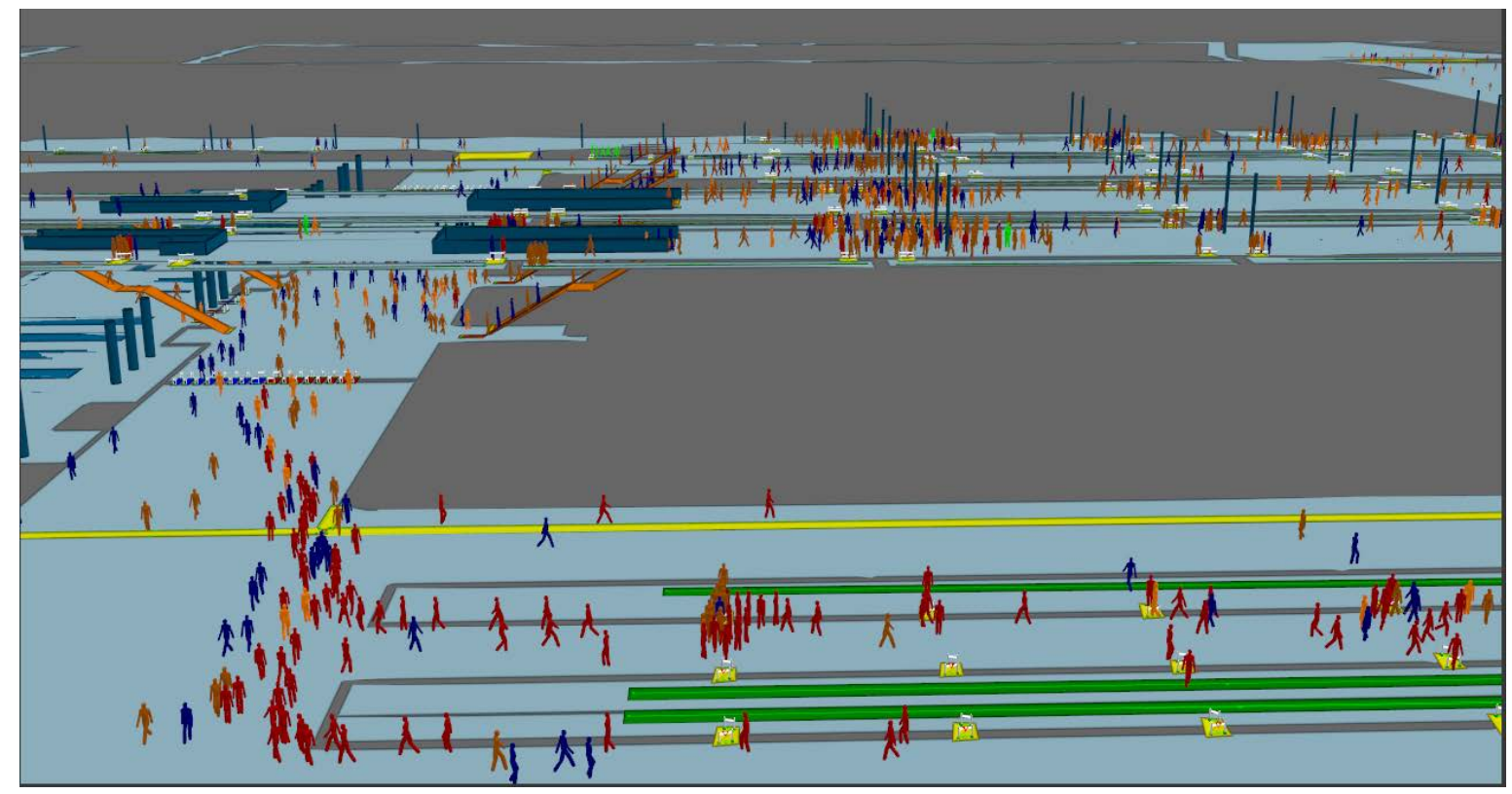

Fig. 4 - Modelización en MassMotion de un intercambiador en Holanda (Arup).

\section{CONCLUSIONES}

El proceso que requiere el diseño de espacios urbanos va más allá del mero cumplimiento de especificaciones técnicas y estándares internacionales. El avance de la técnica en las últimas décadas permite disponer actualmente de ordenadores potentes que realizan en muy poco tiempo complejos análisis dinámicos, mediante el uso de programas informáticos que incluyen en sus algoritmos componentes aleatorias que simulan con gran realismo el movimiento de grandes flujos peatonales en espacios tridimensionales y que poco a poco se está convirtiendo en otra herramienta más integrada en el proceso de concepción de espacios públicos destinados a servir a multitudes. 
Las geometrías de dichos espacios se pueden alterar a conveniencia durante su fase de planificación y diseño, creando diferentes escenarios y diversos tipos de usuarios, permitiendo evaluar multitud de soluciones con todo detalle antes de su construcción, bajo unos parámetros óptimos de seguridad y comodidad para las personas potencialmente usuarias de dicho espacio público. El empleo de una herramienta como MassMotion posibilita la creación de modelos complejos que representan la experiencia del peatón en el espacio urbano que habita. Los espacios bien diseñados, calculados y construidos pueden funcionar como se espera de ellos sin necesidad de efectuar propiamente una modelización peatonal del mismo que lo valide, pero puede que el funcionamiento después no sea el esperado por no considerar aspectos aleatorios propios del libre albedrío sobre los que se basa la naturaleza humana, y de la componente grupal en el comportamiento de grupos de personas, y que actúa de manera concomitante con la propia elección individual de la ruta.

\section{REFERENCIAS}

JOHN J. FRUIN, (1971). Pedestrian Planning and Design. Metropolitan Association of urban designers and enviromental planners, INC.

DIJKSTRA E.W. (1959). A note on two problems in connexion with graphs. Numerische Mathematik 1, pp. 269-271

HELBING D. AND MOLNAR. P. (1995). Social force model for pedestrian dynamics. Phisical Review Volume 51, Number 5. 